This item was submitted to Loughborough's Research Repository by the author.

Items in Figshare are protected by copyright, with all rights reserved, unless otherwise indicated.

\title{
Quasi-flat acoustic absorber enhanced by metamaterials
}

PLEASE CITE THE PUBLISHED VERSION

http://dx.doi.org/10.1121/2.0000010

\section{PUBLISHER}

Acoustical Society of America

VERSION

AM (Accepted Manuscript)

LICENCE

CC BY-NC-ND 4.0

\section{REPOSITORY RECORD}

Ouahabi, Abdelhalim Azbaid El, Victor V. Krylov, and D.J. O'Boy. 2015. "Quasi-flat Acoustic Absorber Enhanced by Metamaterials". figshare. https://hdl.handle.net/2134/18514. 


\title{
(S). Proceedings of Meetings on Acoustics
}

Volume 22

http://acousticalsociety.org/

\section{8th Meeting of the Acoustical Society of America}

Indianapolis, Indiana

27-31 October 2014

Noise: Paper 1aNS3

\section{Quasi-flat acoustic absorber enhanced by metamaterials}

\author{
Abdelhalim Azbaid El Ouahabi, Victor V. Krylov and Daniel J. O'Boy \\ Department of Aeronautical and Automotive Engineering, Loughborough University, Loughborough, \\ Leicestershire, LE113TU, United Kingdom;V.V.Krylov@lboro.ac.uk
}

In this paper, the design of a new quasi-flat acoustic absorber (QFAA) enhanced by the presence of the impedance matching metamaterial layer is described, and the results of the experimental investigation into the reflection of sound from such an absorber are reported. The gradient metamaterial layer is formed by a quasi-periodic array of brass cylindrical tubes with the diameters gradually increasing from the external row of tubes facing open air towards the internal row facing the absorbing layer made of a porous material. The QFAA is placed in a wooden box with the dimensions of 569 x 250 x $305 \mathrm{~mm}$. All brass tubes are of the same length $(305 \mathrm{~mm})$ and fixed between the opposite sides of the box. Measurements of the sound reflection coefficients from the empty wooden box, from the box with an inserted porous absorbing layer, and from the full QFAA containing both the porous layer and the array of brass tubes have been carried out in an anechoic chamber at the frequency range of 500-3000 Hz. The results show that the presence of the metamaterial layer brings a noticeable reduction in the sound reflection coefficients in comparison with the reflection from the porous layer alone.

Published by the Acoustical Society of America 


\section{Introduction}

Acoustic metamaterials represent a relatively new area in applied physics and acoustics that received extensive attention over the last several years, mainly because they can provide acoustic properties that otherwise would be hard or impossible to find in nature. Consequently, they open the door for improved or completely new applications in such areas as damping structural vibrations, sound absorption and noise control. The basic principle of acoustic metamaterials is similar to that of metamaterials in other areas of physics. They usually gain their properties from structure rather than composition, using the inclusion of small inhomogeneities to achieve effective macroscopic behavior [1-4].

As specific examples of metamaterial structures, novel physical objects, "acoustic black holes" in the field of acoustics and "optic black holes" in the field of optics, have been proposed and investigated over the last years. Acoustic black holes have been investigated mainly for flexural waves in thin plates [5-9], for which the required gradual changes in local wave velocity with distance to almost zero according to a power-law profile can be easily achieved by changing the plates' local thickness. A brief review of the recent theoretical and experimental investigations of acoustic black holes for flexural waves can be found in the paper [10]. In the case of electromagnetic metamaterials, optic black holes as structures working as an omnidirectional light absorbers, have been recently designed [11] and tested experimentally $[12,13]$. In particular, it has been shown in [11] that spherical or cylindrical metamaterial layers with dielectric constants altering with distance according to the inverse power law can capture incident light and dissipate it efficiently in the central absorbing core. Note that the principle and the theory of optic black holes [11] are almost identical to those earlier developed for acoustic black holes for flexural waves [5-10].

In contrast to flexural waves in plates, it is much more problematic to slow down acoustic waves propagating in gases or liquids. There are currently two possibilities of creating acoustic black holes for absorption of sound in gases and liquids. The first one is based on the theoretical paper on acoustic black holes for sound absorption in air using wave propagation in a waveguide [14], It was proposed to use an inhomogeneous acoustic waveguide with walls of variable impedance obtained via a quasi-periodic ribbed structure to achieve the required linear decrease in acoustic wave velocity with propagation distance to almost zero. The second possibility is based on gradient metamaterials as wave retarding structures, as was done in the case of optic black holes [11]. However, the required reduction in sound velocity down to almost zero has not been achieved in this way so far. Therefore, in the recent publications the attention has been paid to the design of cylindrical or spherical devices as omnidirectional sound absorbers [15-18] using acoustic metamaterials for gradual impedance matching between the air and the absorbing core.

In the present work, a new "Quasi-Flat Acoustic Absorber” (QFAA) enhanced by the presence of a gradient metamaterial layer for efficient sound absorption in air is designed, constructed and tested. It consists of an absorbing layer and a quasi-periodic array of solid cylinders (brass cylindrical tubes) with their filling fractions varying from the external row facing the open air towards the internal row facing the absorbing layer made of a porous material. A graded metamaterial layer which employs those tubes of varying 
external diameter and spacing is used to gradually adjust the impedance of the air to that of the porous absorbing material and thus to reduce the reflection. Two types of common porous absorbers (Sponge and Fiberglass) are tested in this work to demonstrate the importance of matching the effective acoustic impedance at the exit of the metamaterial layer to that of the porous material in order to ensure maximal absorption into the QFAA. All the brass tubes are of the same length $(305 \mathrm{~mm})$ and arranged as a rectangular array placed into a wooden box with the dimensions of 569 × 250 x $305 \mathrm{~mm}$. The designed structure was manufactured and experimentally tested in an anechoic chamber at the frequency range of $500-3000 \mathrm{~Hz}$.

\section{Experiments}

A wooden box with the dimensions of 569x250x305 mm was designed with two zones, one for the impedance matching metamaterial and the other - for a porous absorbing material.

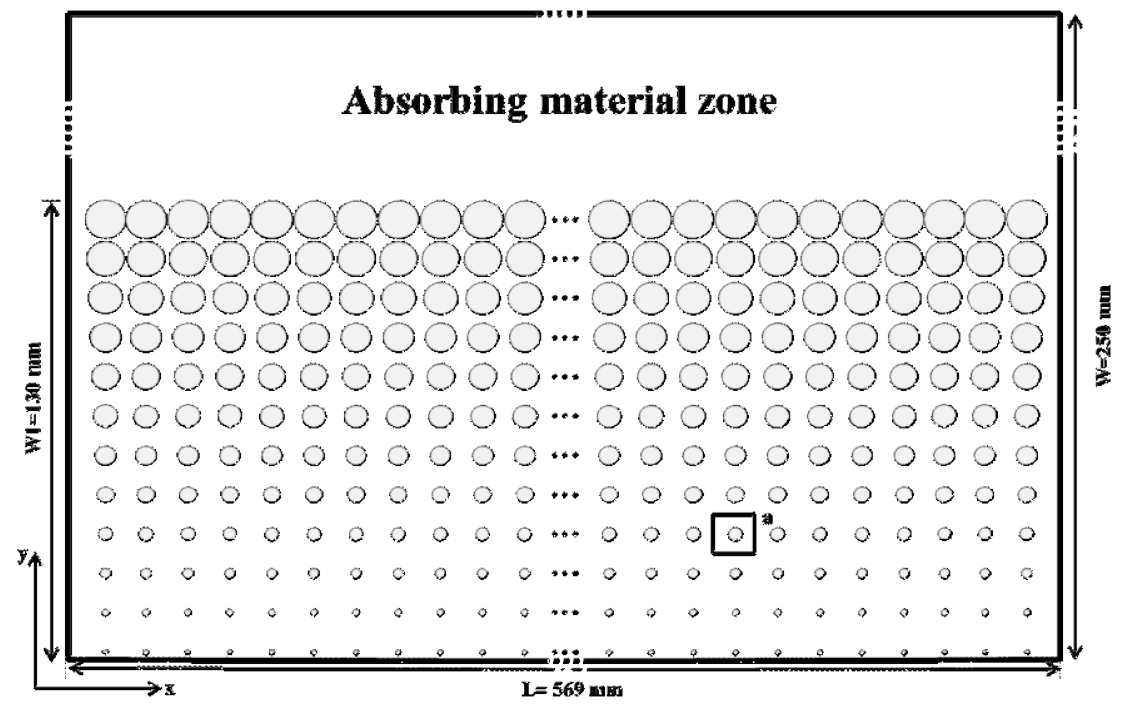

Fig. 1. Schematic top view of the wooden box showing the absorbing material zone and the impedance matching metamaterial zone with the distribution of holes according to a square lattice with the lattice parameter a $=11 \mathrm{~mm}$.

The zone of matching metamaterial was drilled in opposite sides to provide an array of holes with the diameters gradually increasing from the external row facing the open air towards the internal row facing the absorbing material. The holes were arranged in 12x51 pattern with the square lattice constant $\mathrm{a}=11 \mathrm{~mm}$. Figure 1 shows a top-view schematic depicting the distribution of holes on the rectangular board. By inserting $305 \mathrm{~mm}$ long bras cylinders between the opposite-sides of the wooden box into the holes, a Quasi-Flat Acoustic Absorber (QFAA) is constructed as a system of solid cylinders with varying filling fraction backed by the absorbing material. A photograph and a 3D view of the QFAA are shown in Fig. 2(a) and 2(b) respectively. 
(a)

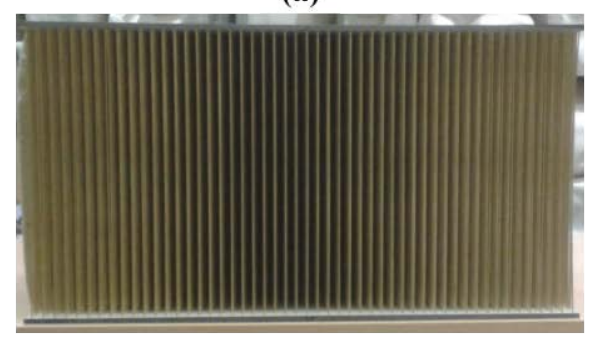

(b)

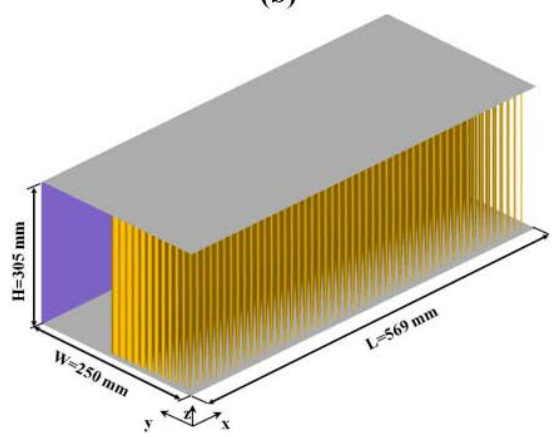

Fig. 2. A frontal photograph (a) and a 3D view (b) of the Quasi-Flat Acoustic Absorber.

Filling fraction $f f$ and effective acoustic impedance $Z_{\text {eff }}$ are defined as follows [17]:

$$
\begin{gathered}
f f=\pi\left(\frac{D}{2 a}\right)^{2} \\
Z_{\text {eff }}=Z_{0} \frac{\sqrt{1+f f}}{1-f f}
\end{gathered}
$$

where $D$ is the diameter of the cylinders and $Z_{0}=413$ Rayl is the impedance of air. Properties of the matching metamaterial layer are summarised in Table 1.

\begin{tabular}{|c|c|c|c|}
\hline $\begin{array}{c}\text { Row } \\
\text { Number }\end{array}$ & $\begin{array}{c}\text { Cylinder } \\
\text { Diameter (mm) }\end{array}$ & $\begin{array}{l}\text { Filling } \\
\text { fraction }\end{array}$ & $\frac{Z_{\text {eff }}}{Z_{0}}$ \\
\hline 1 & 1.6 & 0.0166 & 1.02 \\
\hline 2 & 2.4 & 0.0374 & 1.06 \\
\hline 3 & 3.2 & 0.0665 & 1.11 \\
\hline 4 & 4 & 0.1039 & 1.17 \\
\hline 5 & 4.8 & 0.1496 & 1.26 \\
\hline 6 & 5.6 & 0.2036 & 1.38 \\
\hline 7 & 6.3 & 0.2576 & 1.51 \\
\hline 8 & 7.1 & 0.3272 & 1.71 \\
\hline 9 & 7.9 & 0.4051 & 1.99 \\
\hline 10 & 8.7 & 0.4913 & 2.4 \\
\hline 11 & 9.5 & 0.5858 & 3.04 \\
\hline 12 & 10.3 & 0.6886 & 4.17 \\
\hline & & & \\
\hline
\end{tabular}

Table 1. Properties of the impedance matching metamaterial layer formed by the array of brass cylinders. 
The calculated effective impedance defined by equation (2) and normalized to the impedance of air is plotted in Fig. 3 as a function of a row number.

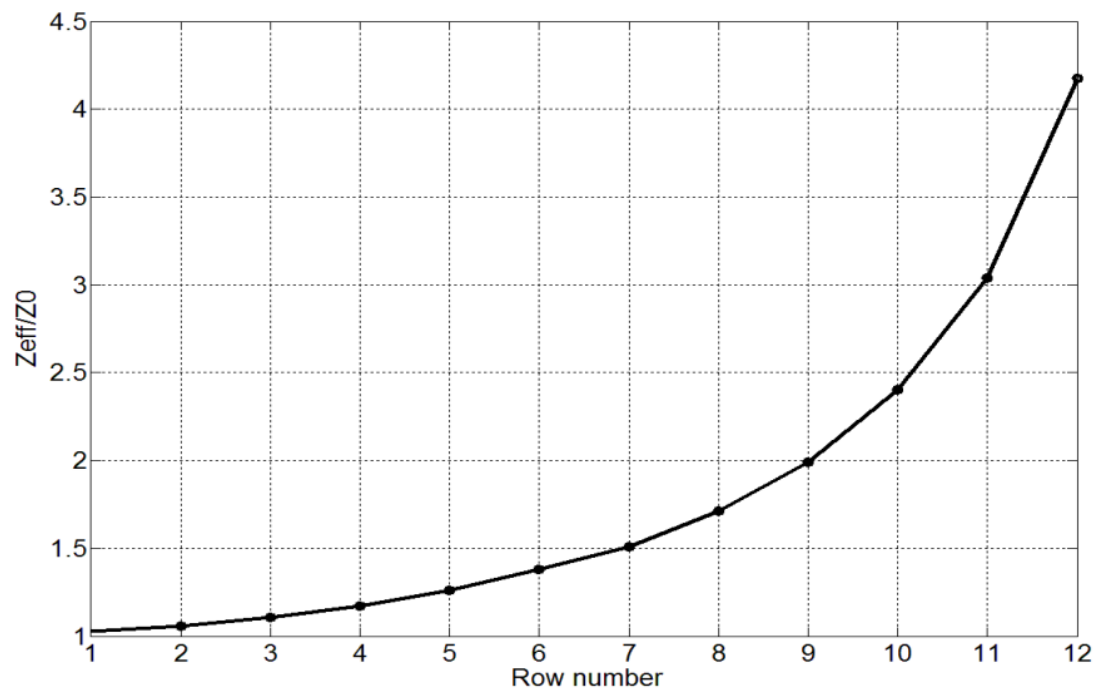

Fig. 3. Normalized effective impedance of the metamaterial layer as a function of a row number.

The experiments have been carried out in the anechoic chamber of the Department of Aeronautical and Automotive Engineering at Loughborough University. The walls, ceiling and floor of the chamber are lined with glass-fiber wedges $(\mathrm{G}+\mathrm{H}$ ASONAD) that provide sound absorption at frequencies above $100 \mathrm{~Hz}$ and thus prevent sound reflection from the room boundaries, so that free-field conditions hold. The effective dimensions between wedges are: $6.4 \times 5.2 \times 5.3 \mathrm{~m}$, and the acoustically usable 'free field' range dimensions are: 4.7 x 3.5 x $3.6 \mathrm{~m}$.

As a sound source we used a loudspeaker suspended on a pulley system that allowed its center to be located at $853 \mathrm{~mm}$ height above ground. The centre of QFAA surface was aligned with the loudspeaker and placed at $2 \mathrm{~m}$ from the source in order to produce the desired almost plane wave fronts when the sound reaches the sample. Additional glassfiber wedges were placed on the ground in the area of the measurement and on front of the recording unit to avoid undesired reflection of sound. Two type of G.R.A.S. 40AE, pre-polarized $1 / 2$ inch free-field microphones (their sensitivities are $44 \mathrm{mV} / \mathrm{Pa}$ (Mic1) and $42 \mathrm{mV} / \mathrm{Pa}$ (Mic2)), with G.R.A.S. preamplifiers type 26CA, were used to measure sound pressure. Figure 4 shows the photograph of the experimental set up utilised to measure the sound pressure reflection coefficients. 


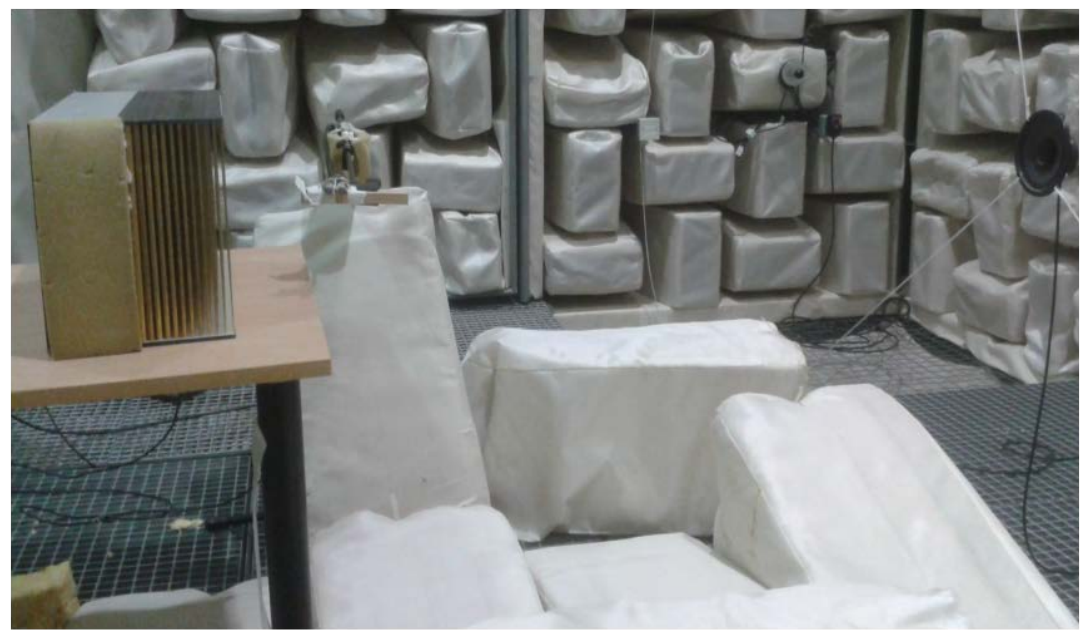

Fig. 4. Photograph of the experimental set up showing the QFAA (left) and the loudspeaker (right).

Two methods of measuring the sound pressure reflection coefficients have been used: the traditional Standing Wave Ratio method (SWR) and the Transfer Function Method (TFM). Schematic view of the experimental setups using SWR method and TFM method are shown in Fig. 5, (a) and (b) respectively.

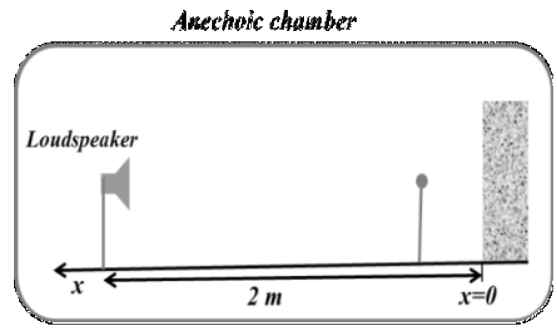

(a)

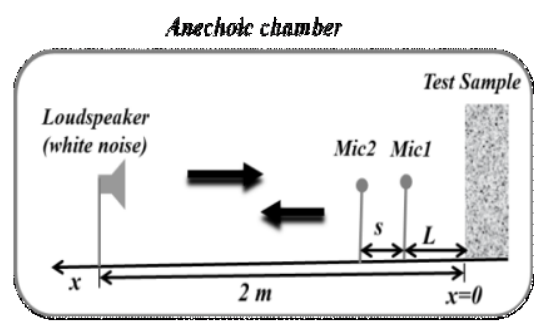

(b)

Fig. 5. Schematic view of the experimental setups: (a) Standing Wave Ratio Method; (b) Transfer Function Method.

Let us discuss first the SWR method (Fig. 5(a)). If plane waves are assumed to propagate in front of a sample, then the superposition of the incident and reflected waves is given by:

$$
p=A\left(e^{i k x}+R e^{-i k x}\right),
$$

where $R$ is the reflection coefficient; $k$ is the wavenumber, and $A$ is a complex constant; the sample is assumed to be at $x=0$. The first term represents the incident wave 
and the second - the reflected wave. The maximum pressure $P_{\max }$ occurs when the first and second terms in Eq. (3) are in phase, and the minimum pressure $P_{\min }$ occurs when they are out of phase. The standing wave ratio $S$, which is defined as the ratio between the maximum pressure and the minimum pressure, can be expressed as a function of the absolute value of the reflection coefficient $|R|$ :

$$
S=\frac{p_{\max }}{p_{\min }}=\frac{1+|R|}{1-|R|} .
$$

This formula can be rearranged to allow the magnitude of the reflection coefficient to be obtained:

$$
|R|=\frac{p_{\max }-p_{\min }}{p_{\max }+p_{\min }} .
$$

Samples were placed at $2 \mathrm{~m}$ from the source in order to produce the desired planewavefronts at the samples' location. Then the expression (5) has been applied. A signal generator was used to input frequencies from 500 to $3000 \mathrm{~Hz}$ to produce sine waves by the loudspeaker. A single microphone was used to measure the maximum and minimum values of the sound pressure near the front of the sample, and then the reflection coefficient was calculated.

Let us now briefly discuss the TFM method (Fig. 5(b)), assuming again the generation of plane waves in front of the sample (see also [19]). Equation (3) has two unknowns: the magnitude and phase of the reflection coefficient. By measuring acoustic pressure $p$ at two points in close proximity of the sample and calculating the transfer function between two microphone positions

$$
H_{21}=\frac{p\left(x_{1}\right)}{p\left(x_{2}\right)}
$$

it is possible to set up and solve simultaneous equations for the reflection coefficient.

Using equation (3), the transfer function between Mic2 and Mic1 can be written as

$$
H_{21}=\frac{e^{i k x_{1}}+R e^{-i k x_{1}}}{e^{i k x_{2}}+R e^{-i k x_{2}}},
$$

where $x_{1}$ and $x_{2}$ are the positions of the microphones shown in Fig. 5(b).

Rearrangement then leads to the complex pressure reflection coefficient:

$$
R=\frac{H_{21}-e^{-i k s}}{e^{i k s}-H_{21}} e^{i 2 k(L+s)},
$$

where $s$ is the distance between the microphones and $L$ is the distance from the sample face to the Mic1. There are restrictions on the microphone spacing [19]. The lower and upper frequency limits are given by:

$$
\frac{0.05 c}{s}<f<\frac{0.45 c}{s}
$$

where $c$ is the sound velocity. 
A white noise generator was used to drive the loudspeaker. Two microphones have been used to measure sound pressure, and a program compiled in Matlab was used to compute the transfer function between two microphone positions and then calculate the reflection coefficient from the sample.

The distance from the sample face to the first microphone was $155 \mathrm{~mm}$, and the distance between the microphones was $35 \mathrm{~mm}$. The microphones were connected to a PC via a five-channel dynamic signal acquisition module NI-USB-4431 card. Schematic views of the experimental setups using SWR method and TFM method are shown in Fig. 5, (a) and (b) respectively.

\section{Results and discussion}

The choice of porous materials for the absorbing zone was based on values of their acoustic impedance in comparison with the effective impedance at the internal row of the metamaterial quasi-periodic structure described in the previous sections. Two types of absorbing porous materials, sponge and fiberglass, have been used in the absorbing material zone. Impedance measurements have been carried out using the Transfer Function Method. The normalized acoustic impedances of sponge and fiberglass (relative to the acoustic impedance of air $Z_{0}$ ) calculated from the measured reflection coefficients are shown in Fig. 6 as functions of frequency.

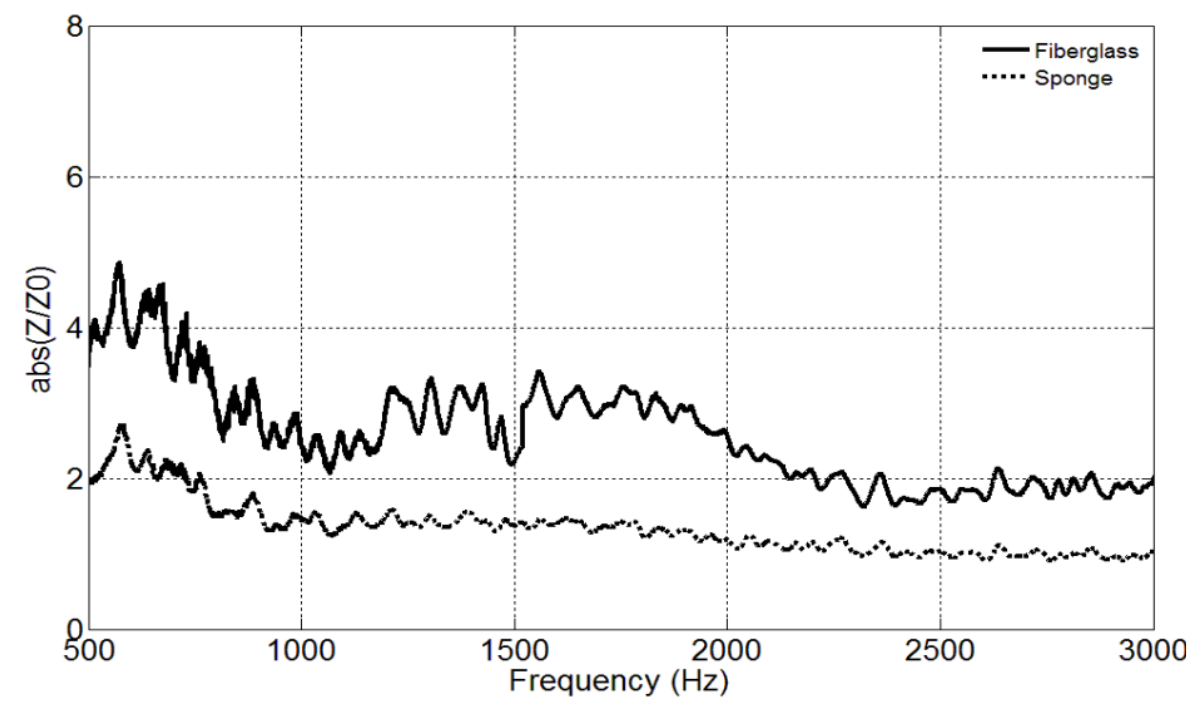

Fig. 6. Normalised acoustic impedances of fiberglass (solid line) and sponge (dotted line) calculated from the measured reflection coefficients.

In the first step of the detailed measurements of the reflection coefficients for the full QFAA and for its different components, the SWR method has been employed to measure the sound pressure as a function of distance from the empty wooden box, from the rigid board, from the box with sponge inserted, and from the full QFAA containing both the sponge and the array of brass tubes at the frequency range of 500 $-3000 \mathrm{~Hz}$. Figure 7 
shows the sound pressure as a function of distance from the full QFAA at the frequencies 500, 1000, 1500 and $3000 \mathrm{~Hz}$.
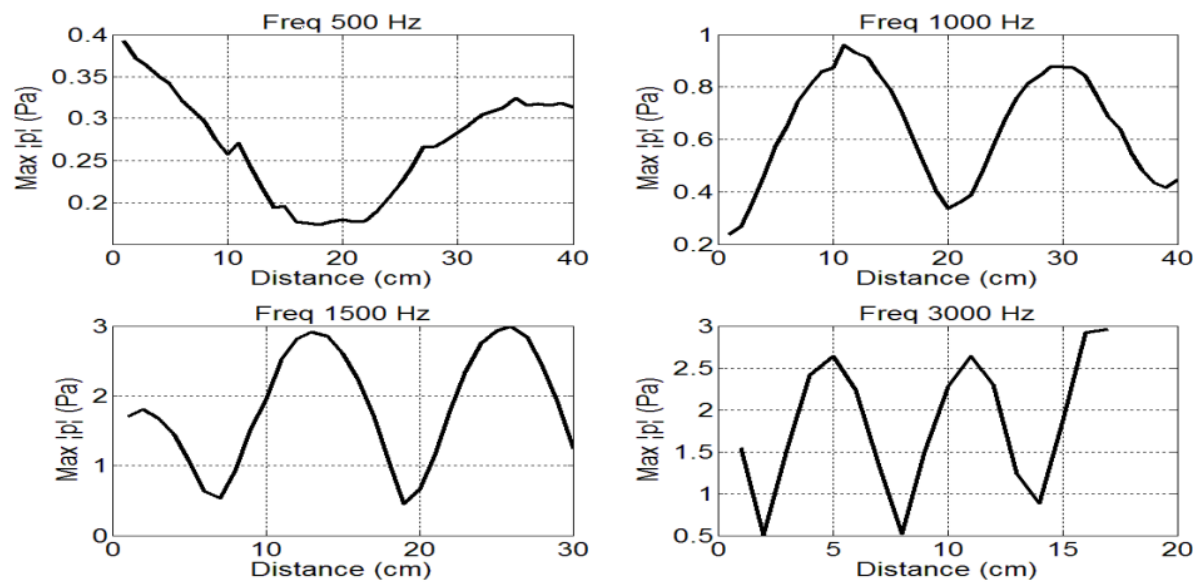

Fig 7. Sound pressure at four fixed frequencies (500, 1000, 1500 and $3000 \mathrm{~Hz}$ ) as a function of distance from the full QFAA with inserted sponge.

As it can be seen from Fig. 7, the sound pressure measured along the $x$-axis in front of the full QFAA shows distinctive standing wave patterns. Therefore, it is easy to determine the sound reflection coefficients by measuring the maximum and minimum values of the sound pressure, using equation (5). Plots of the sound reflection coefficients as functions of frequency obtained by SWR method for the full QFAA with inserted sponge and for some of its components are shown in Fig. 8.
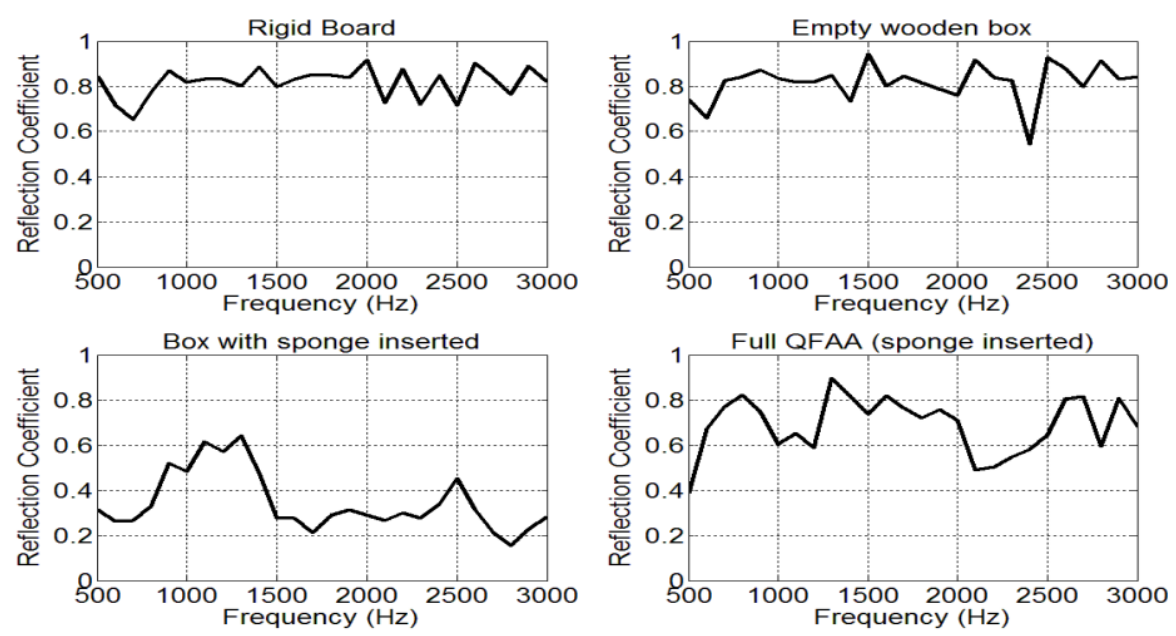

Fig 8. Sound reflection coefficients as functions of frequency obtained by the SWR method for a rigid board, for an empty wooden box, for a box with a sponge, and for the full QFAA. 
Note that the plots in Fig. 8 show that the full QFAA produces lower reflection coefficient in comparison with the reflection from the rigid board and from the empty wooden box. However, it shows higher reflection coefficient than the box with sponge only. These observations illustrate the fact that in this case there is no impedance matching between the sponge and the internal row (row 12) of the metamaterial layer (compare Fig. 3 and Fig. 6).

The same measurements of the reflection coefficients have been repeated with the TFM method for frequencies from $500 \mathrm{~Hz}$ to $3000 \mathrm{~Hz}$. The results are shown in Fig. 9. A comparison between reflection coefficient behaviour measured using the SWR method (Fig. 8) and the TFM method (Fig. 9) shows that the results are quite similar. The TFM method exhibits the same features as the SWR method does. In particular, the box with sponge inserted still produces a lower reflection coefficient than the full QFAA.

In order to achieve lower reflection from the full QFAA described above, one has to insert a suitable porous absorbing material with a matching impedance. In what follows, only the TFM method will be employed to measure the reflection coefficients. The reason for that is that it gives the reflection coefficient from a sample for all frequencies (within limits) using only a couple of quick measurements. In contrast, the SWR method only gives the result at one frequency at a time, and the procedure for locating minima and maxima in the standing wave, which is needed to get the phase information, is rather slow, so that measuring at a large number of frequencies is very time consuming.
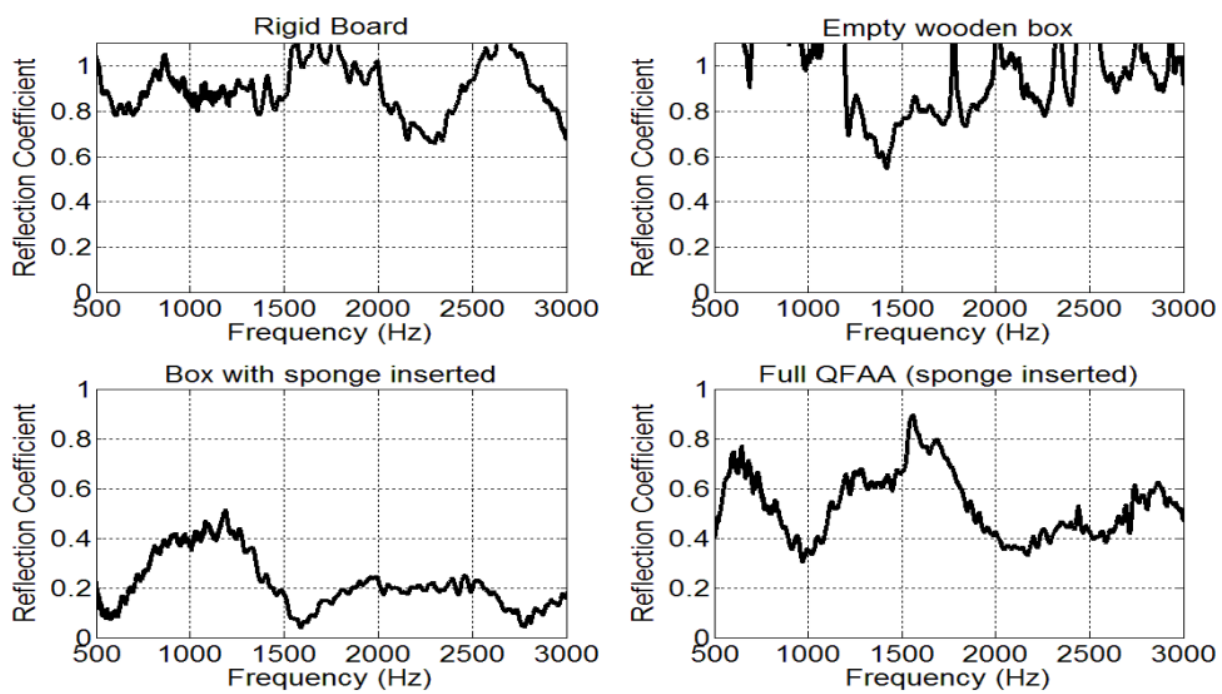

Fig. 9. Sound reflection coefficients as functions of frequency obtained by the TFM method for a rigid board, for an empty wooden box, for a box with a sponge, and for the full QFAA.

The same tests as above were repeated for fiberglass as an absorbing porous material. Its acoustic impedance is greater than that for the sponge, see Fig. 6, so that one would expect its better matching to the effective impedance at the last row of the metamaterial 
layer. The results of the measurements of the reflection coefficients, at frequencies from $500 \mathrm{~Hz}$ to $3000 \mathrm{~Hz}$, for the box with inserted fiberglass and for the full QFAA (the quasiperiodic array of cylinders with fiberglass inserted) are shown in Fig. 10. It can be seen that at the frequency range of $500-1581 \mathrm{~Hz}$ and of $2434-2745 \mathrm{~Hz}$, the QFAA with fiberglass inserted provides lower reflection coefficient than the box with fiberglass inserted. In addition, it also provides lower reflection coefficient than the QFAA with sponge inserted (see Fig. 9)), except for the frequency range of 1920 - $2432 \mathrm{~Hz}$.

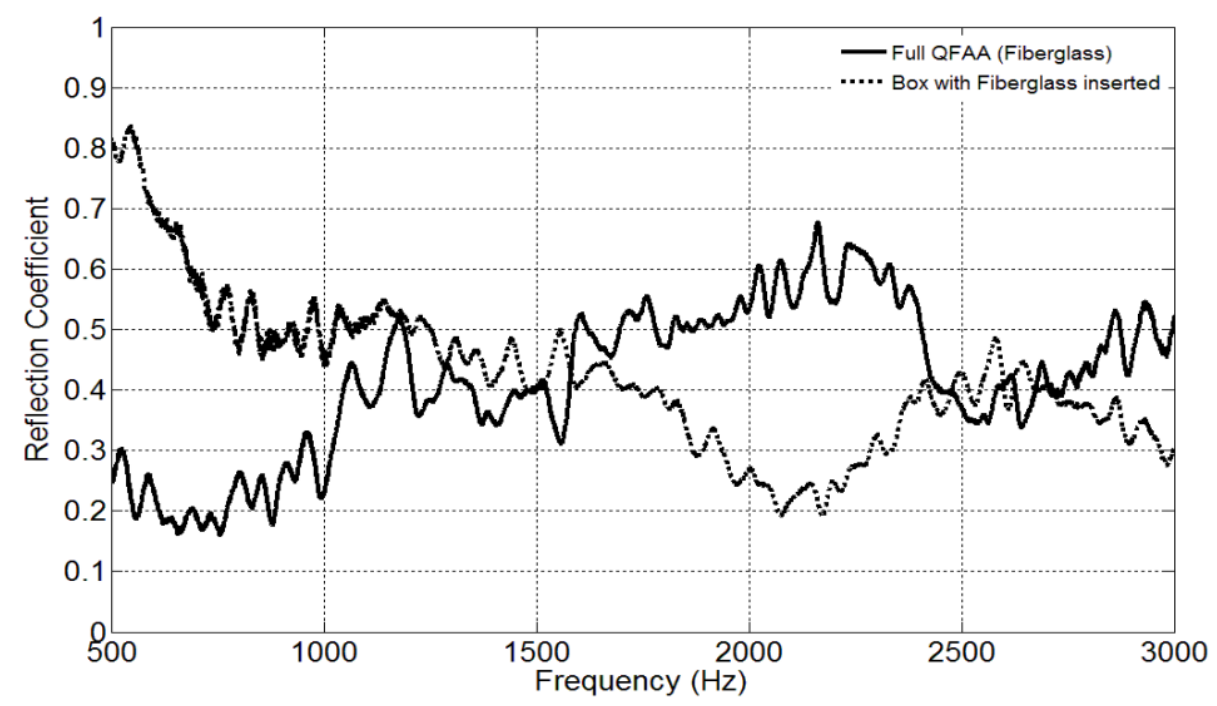

Fig. 10. Frequency dependence of the sound reflection coefficients for a box with fiberglass and for the full QFAA with 12 rows of brass cylinders and with fiberglass inserted; measurements made using TFM method.

Although the measured reflection coefficients in Fig. 10 clearly demonstrate the benefit of using a matching metamaterial layer to reduce reflection, further improvement can be made. In order to achieve better results, one needs to adjust the effective acoustic impedance at the exit of the metamaterial layer to make it even closer to the acoustic impedance of the inserted fiberglass. Therefore, it has been decided to remove a few last rows of brass cylinders to reduce the effective impedance at the exit of the metamaterial layer and to get an adequate matching of the impedances. Two last rows have been removed, thus reducing the relative effective impedance at the exit of the matching metamaterial layer down to 2.4 .

Measurement of the reflection coefficient have been carried out for the full QFAA containing only 10 rows of brass cylinders, with fiberglass inserted. The results are shown in Fig. 11 in comparison with the results for the box with fiberglass inserted, but in the absence of the metamaterial layer. It can be seen that at all frequencies the reflection coefficient for the box with fiberglass inserted is strongly reduced when the QFAA (with 10 rows of brass cylinders and with fiberglass inserted) has been added. This demonstrates the functionality of matching the impedances using metamaterial layers. The device with 10 rows strongly outperforms the device with 12 rows in terms of the values of reflection coefficient at frequencies above $750 \mathrm{~Hz}$. However, it provides higher 
reflection coefficient for frequencies below $750 \mathrm{~Hz}$. Note that at frequencies above 750 $\mathrm{Hz}$ the values of reflection coefficient do not exceed $26 \%$. This means that at these frequencies the full QFAA with 10 rows of solid cylinders acts as an efficient acoustic absorber, with more than 93\% of the impinging acoustic energy being absorbed.

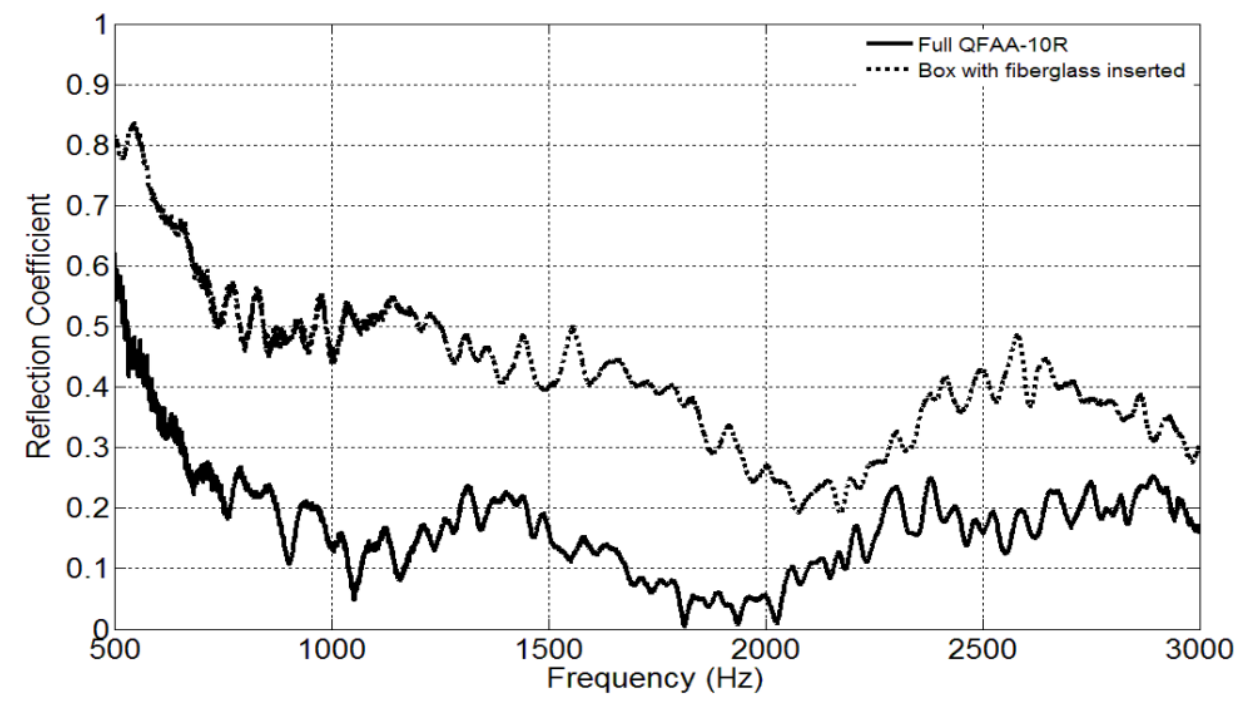

Fig. 11. Sound reflection coefficients measured for the box with fiberglass inserted (dotted line) and for the full QFAA with 10 rows of brass cylinders and fiberglass inserted (solid line); measurements made using TFM method.

\section{Conclusions}

A quasi-flat acoustic absorber (QFAA) enhanced by the presence of a gradient metamaterial layer has been designed, manufactured and tested over a wide range of frequencies. The QFAA consists of a quasi-periodic system of solid cylinders with varying filling fraction and an absorbing layer made of a porous material. The impedance matching metamaterial layer was formed by up to twelve rows of brass cylinders of equal length and with diameters gradually increasing from the external row facing the open air towards the internal row facing the absorbing layer.

It has been demonstrated experimentally that the values of sound reflection coefficient for the QFAA depend strongly on the impedance matching between the porous absorbing material and the exit of the gradient metamaterial layer. In particular, it has been shown that the full QFAA with 10 rows of cylinders and with fiberglass as inserted absorbing material is more efficient than the full QFAA with 12 rows of cylinders (also with fiberglass inserted). This can be explained by a nearly perfect impedance matching achieved in this case. The obtained results show that, for the quasi-flat geometrical configuration considered, the presence of the impedance matching metamaterial layer in 
front of the porous absorbing material can bring a substantial reduction in sound reflection coefficient in comparison with the case of reflection from the porous material alone.

\section{Acknowledgements}

The research reported here has been supported by EPSRC grant EP/K038214/1.

\section{References}

[1] N. Engheta and R.W. Ziolkowski, "Metamaterials: physics and engineering explorations". Wiley \& Sons. Chapter 1, 2006.

[2] V.G. Veselago, "The electrodynamics of substances with simultaneously negative values of $\varepsilon$ and $\mu$ ”. Sov. Phys. Uspekhi, vol. 10, 509-514, 1968.

[3] R.A. Shelby, D.R. Smith and S. Schultz, "Experimental verification of a negative index of refraction”, Science, vol. 292, 77-79, 2001.

[4] J.B. Pendry, "Negative refraction”, Contemporary Phys, vol. 45, 191-202.

[5] V.V. Krylov, "Laminated plates of variable thickness as effective absorbers for flexural vibrations," in Proc. $17^{\text {th }}$ Int. Congress on Acoustics, 2001, vol. 1, pp. 270271.

[6] V.V Krylov, "New type of vibration dampers utilising the effect of acoustic 'black holes'," Acta Acust. united with Acust., vol. 90, no. 5, pp. 830-837, 2004.

[7] V.V. Krylov and F.J.B.S. Tilman, "Acoustic 'black holes' for flexural waves as effective vibration dampers,” J. Sound and Vibr., vol. 274, no. 3-5, pp. 605-619, 2004.

[8] V.V. Krylov and R.E.T.B. Winward, "Experimental investigation of the acoustic black hole effect for flexural waves in tapered plates, J. Sound and Vibr., vol. 300, no. 1-2, pp. 43-49, 2007.

[9] E.P. Bowyer, D.J. O'Boy, V.V. Krylov, and F. Gautier, "Experimental investigation of damping flexural vibrations in plates containing tapered indentations of power-law profile", Applied Acoustics, vol. 74, no. 4, pp. 553-560, 2013.

[10] V.V. Krylov, "Acoustic black holes: Recent developments in the theory and applications,” IEEE Trans. on Ultrasonics, Ferroelectrics, and Frequency control,” vol. 61, no. 8, pp. 1296-1306, 2014.

[11] E.E. Narimanow and A.V. Kildishev, "Optical black hole: Broadband omnidirectional light absorber”. Appl. Phys. Lett. vol. 95, 041106, 2009.

[12] Q. Cheng, T.J. Cui, W.X. Jiang, and B.G. Cai, “ An omnidirectional electromagnetic absorber made of metamaterials,” New J. Phys. vol. 12, 063006, 2010.

[13] J. Zhou, X. Cai, Z. Chang, and G. K. Hu, " Experimental study on a broadband omnidirectional electromagnetic absorber,” J. Opt. vol. 13, 085103, 2011.

[14] M.A. Mironov and V.V. Pislyakov, "One-dimensional acoustic waves in retarding structures with propagation velocity tending to zero," Acoust. Phys. vol. 48, no. 3, pp. 347-352, 2002.

[15] R. Li, X. Zhu, B. Liang, Y. Li, X. Zou, and J. Cheng, “ A broadband acoustic omnidirectional absorber comprising positive index materials,” Appl. Phys. Lett. vol. 99, 193507, 2011. 
[16] A. Climente, D. Torrent, and J. Sanchez-Deseha, "Omnidirectional broadband acoustic absorber based on metamaterials,” Appl. Phys. Lett. vol. 100, 144103, 2012.

[17] O. Umnova and B. Zajamsek, " Omnidirectional graded index sound absorber”, in Proc. Conf. Acoustics, pp. 3631-3637, 2012.

[18] C.J. Naify, T.P. Martin, C.N. Layman, M. Nicholas, A.L. Tangawng, D.C. Calvo, and G.J. Orris, "Underwater acoustic omnidirectional absorber," Appl. Phys. Lett. vol. 104, 073505, 2014.

[19] ISO 10534-2, Acoustics - Determination of sound absorption coefficient and impedance in impedance tube - Part 2: Transfer-function method. 